\title{
Physical exercise and migraine: for or against?
}

\author{
Giuseppe Lippi ${ }^{1}$, Camilla Mattiuzzi ${ }^{2}$, Fabian Sanchis-Gomar ${ }^{3,4}$ \\ ${ }^{1}$ Section of Clinical Biochemistry, University of Verona, Verona, Italy; ${ }^{2}$ Service of Clinical Governance, Provincial Agency for Sanitary Services, \\ Trento, Italy; ${ }^{3}$ Leon H. Charney Division of Cardiology, New York University School of Medicine, New York, USA; ${ }^{4}$ Department of Physiology, \\ Faculty of Medicine, University of Valencia and INCLIVA Biomedical Research Institute, Valencia, Spain \\ Correspondence to: Prof. Giuseppe Lippi. Section of Clinical Biochemistry, University Hospital of Verona, Piazzale LA Scuro, 37100 Verona, Italy. \\ Email: giuseppe.lippi@univr.it.
}

Submitted Mar 26, 2018. Accepted for publication Apr 10, 2018.

doi: $10.21037 / \mathrm{atm} .2018 .04 .15$

View this article at: http://dx.doi.org/10.21037/atm.2018.04.15

\section{Introduction}

Migraine can now be considered a major public healthcare issues, since it is one of the leading causes of disability worldwide, causing a considerable number of clinical, social and economic issues (1). Recent statistics attest that the prevalence of this condition may be as high as $25 \%$ in adults, with approximately $4 \%$ of the general population suffering from a chronic form of disease. Albeit the precise pathogenesis of migraine still remains substantially unknown (2), many effective therapeutic strategies have been proposed, mainly entailing the administration (alone or in combination) of triptans, ergots, acetaminophen and nonsteroidal anti-inflammatory drugs (NSAIDs). Besides, there is still uncertainty of the real effectiveness in many migraine patients of some possible prophylactic or preventive measures encompassing drugs, lifestyle changes and dietary habits (1).

The relationship between physical exercise and migraine remains rather controversial. On one hand, worsening of migraine pain has often been perceived by patients during or after regular physical exercise $(3,4)$. However, in a critical review of scientific literature published nearly 10 years ago, Busch and Gaul concluded that although the data available at that time was mostly insufficient for establishing whether or not exercise may be an effective therapeutic or preventive measure for improving migraine symptoms and quality of life of affected patients, an active lifestyle is often recommended by many migraine experts (5). Ten years afterward, many and more recent epidemiologic and intervention studies were published, which should actually provide an updated and more straightforward contribution to dispel the doubts about the intriguing relationship between physical exercise and migraine. Therefore, the aim of this article is to provide a narrative review of recent scientific articles (i.e., published from the year 2008 to present time) which have explored the potential association between physical exercise and migraine.

\section{Epidemiologic studies}

Varkey et al. carried out a large prospective study including 22,397 participants to investigate the potential relationship between physical exercise and migraine (6). After 11 years of follow-up, a light physical exercise (i.e., no sweating or being out of breath) was associated with a $35 \%$ enhanced risk of migraine (OR, 1.35; 95\% CI, 1.21-1.51) compared to hard physical exercise (i.e., sweating or being out of breath).

Milde-Busch et al. administered a lifestyle questionnaire to 1,260 adolescents to identify potential correlates of migraine (7). These authors found that a low level of metabolic equivalent index of physical exercise was associated with a 4.2-fold higher risk of migraine compared with to a high level of metabolic equivalent index [odds ratio (OR), 4.2; 95\% confidence interval (95\% CI), 2.2-7.9].

Winter et al. reported the results of a large population study based on 7,417 individuals, in which the potential relationship between many lifestyle factors and migraine was investigated (8). No association was observed between the level of physical exercise and the risk of developing migraine attacks.

In a subsequent article, Winsvold et al. published the 
results of the Nord-Trøndelag Health Study in Norway 1995-1997 (HUNT 2) study, in which 48,713 subjects aged 20 years or older were administered with a questionnaire on potential lifestyle factors associated with development of migraine (9). Compared to patients without migraine $(63.6 \%)$, those with $(68.7 \%)$ or without $(68.4 \%)$ aura reported a significantly higher burden of physical inactivity.

Koppen et al. interviewed 103 migraine patients first presenting at a headache clinic to identify potential correlates of migraine (10). Overall, 39/103 patients (38\%) reported a risk of developing migraine attacks during exercise, most of which during endurance activities (i.e., running). All of these patients also reported that migraine attacks tended to worsen by practicing physical exercise. Interestingly, neck pain and headaches were both more frequent in patients with exercise-provoked migraine than in those without.

Lebedeva et al. performed a large population study, including 3,124 subjects ( $43 \%$ women) who were interviewed with the purpose of identifying potential lifestyle factors associated with the risk of developing migraine (11). In multivariate logistic regression analysis, low physical exercise (i.e., $<30 \mathrm{~min}$ of physical exercise per week) was associated with a $60 \%$ higher risk of developing migraine (OR, 1.6; 95\% CI, 1.0-2.4). Notably, this association was found to be statistically significant in women (OR, 1.9; 95\% CI, 1.1-3.2) but not in men (OR, 1.2; 95\% CI, 0.6-2.6).

Krøll et al. performed a population study based on 148 patients followed at a tertiary specialized migraine center and 100 healthy matched controls, who were interviewed about their lifestyle factors (12). The risk of having a migraine was hence found to be 4.4-fold higher (OR, 4.4; $95 \%$ CI, 1.6-11.7) for subjects with a low level of physical exercise ( $<30 \mathrm{~min}$ of moderate-intensity physical exercise on most days) compared to those with a high level ( $\geq 1$ hour per day or more of moderate-intensity physical exercise).

Similar data was reported in a subsequent study by Farris et al., who administered a physical activity questionnaire to 132 migraine women aged between 18-50 (13). According to the results of the surveys, no significant variation of pain while performing physical activity was reported in the vast majority of participants (i.e., $62 \%$ ), worsened pain was reported by $35 \%$ of participants, whilst symptoms improvement was only noticed by $3 \%$ of participants. Notably, physical activity led to pain worsening during all attacks in only $10 \%$ of participants.
Hagen et al. carried out a population-based historical cohort study (Nord-Trøndelag Health Study) to investigate the impact of different lifestyle aspects on the risk of developing migraine (14). The study population consisted of 15,276 participants without migraine at baseline, who were then evaluated over a period of 11 years. Interestingly, 1 to 3 hours of low-intensity physical exercise per week and 1 to 2 hours of hard physical exercise per week were respectively associated with a $22 \%$ [relative risk (RR), 0.78; $95 \%$ CI, 0.62-0.99] and 29\% (RR, 0.71; 95\% CI, 0.54-0.94) lower risk of developing migraine compared to inactivity. A trend towards lower risk of developing migraine was also observed for $\geq 3$ hours of high-intensity physical exercise per week, but the association did not reach statistical significance (RR, 0.92; 95\% CI, 0.67-1.27).

In another study, Luedtke et al. explored surface electromyography responses of trapezius muscle in 28 healthy subjects as well as in 31 patients with chronic migraine and 43 with episodic migraine (15). Increased bilateral trapezius muscle activity was recorded in all groups during physical exercise, but no statistically significant difference could be observed $(\mathrm{P}<0.05)$. It could hence be concluded that trapezius muscle activity should not be considered an underlying trigger but rather should be interpreted as an accompanying symptom in migraineurs.

\section{Intervention studies}

Albeit published before 2008, the article of Narin et al. deserves to be mentioned, since is it one of most accurate studies exploring the effect of physical exercise on migraine (16). Briefly, 40 migraine women were followed by the neurology department and were equally randomized to an exercise group undertaking 1-hour aerobic exercise 3 times weekly and to a control group. After 8 weeks, the intervention group had a substantial improvement of all migraine features, thus displaying a reduction of pain intensity $(-55 \%$ versus pre-exercise and $-43 \%$ versus control group), pain duration (-67\% versus pre-exercise and $-58 \%$ versus control group), pain frequency ( $-51 \%$ versus pre-exercise and $-49 \%$ versus control group) and pain disability index $(-56 \%$ versus pre-exercise and $-51 \%$ versus control group).

In a subsequent study, Dittrich et al. equally randomized 30 migraine women to an aerobic exercise group (1-hour of twice-weekly indoor aerobic exercise program for 6 weeks) and a control group (no change of previous 
physical exercise level) (17). After the intervention period a substantial reduction of intense migraine pain $(-80 \%$ versus pre-exercise and $-75 \%$ versus control group) and frequency of migraine attacks ( $\geq 1$ per week; $-50 \%$ versus pre-exercise and $-67 \%$ versus control group) could be recorded. Interestingly, nearly $90 \%$ of patients in the exercise group perceived that physical exercise was helpful to ameliorate active behavior.

Varkey et al. carried out an intervention study based on 26 migraine patients and entailing $40 \mathrm{~min}$ indoor cycling 3 times weekly for 12 weeks (18). At the end of treatment, a substantial improvement could be observed, as attested by a reduction of pain intensity $(-18 \%)$, migraine attacks per month $(-23 \%)$, days with migraine $(-28 \%)$ and use of antimigraine drugs $(-24 \%)$. Notably, the perceived quality of life was also significantly improved after treatment $(+12 \%)$.

Pairo and collaborators preformed a random semiexperimental study including 20 migraine women (19). The women were randomized into an exercise group (i.e., $40 \mathrm{~min}$ of moderate intensity aerobic exercise 3 times weekly) and a control group (no exercise). One month after the intervention, frequency, intensity and duration of migraine attacks were found to be substantially reduced.

Varkey and coworkers carried out another study based on a specific subset of 14 migraine patients, reporting that physical exercise often led to pain worsening during a migraine attack (20). All patients underwent two exhaustion cycle ergometer tests. Overall, a migraine attack developed after both tests in $3 / 14$ patients (21\%), after one test in 5/14 patients (36\%), whilst 6/14 patients (43\%) did not develop a migraine attack after either trial. Notably, a modest but significant association $(\mathrm{P}=0.036)$ was found between testprovoked migraine and baseline frequency of migraine attacks.

Hanssen et al. performed a controlled randomized trial involving episodic migraine patients, who were randomized into three groups, i.e., high-intensity interval training (HIT; 4 series of 1 min treadmill exercise at heart rate between 90-95\% followed by $3 \mathrm{~min}$ treadmill exercise at target heart rate of $70 \%$, twice weekly), moderate continuous exercise training (MCT; 45 min treadmill exercise at target heart rate of $70 \%$, twice weekly) and no exercise (21). After a 12-week period, both types of exercise were associated with a reduced frequency of migraine attacks, although HIT seemed globally more efficient $(-63 \%)$ than MCT $(-26 \%)$.

Interesting findings emerged from a recent intervention study, in which 52 migraine patients were equally randomized into an exercise group (i.e., $45 \mathrm{~min}$ of aerobic exercise 3 times weekly for 3 months) and a control group (i.e., usual daily activities, no aerobic physical exercise) (22). After the end of the study and during 6 months of follow-up, the aerobic exercise group displayed a substantial improvement of all the main migraine features, i.e., reduction of days with migraine $(-23 \%$ versus both pre-exercise and control group), pain intensity $(-16 \%$ versus pre-exercise and $-8 \%$ versus control group) and pain duration $(-28 \%$ versus pre-exercise, no difference with control group). Even more importantly, the quality of life of the exercise group was substantially improved during follow-up, as shown by a remarkable increase in the time spent on household chores $(+32 \%)$, paid work or study tasks $(+46 \%)$, social activities $(+31 \%)$, family life $(+33 \%)$, and physical exercise $(+17 \%)$.

\section{Conclusions}

The current available evidence garnered from the respectable number of recent epidemiologic investigations and intervention studies would lead us to formulate a number of evidence-based conclusions. Indeed, the results of most recent epidemiological studies are consistent to show an inverse association between physical exercise and migraine, but it is quite challenging to establish whether physical inactivity may be the cause or the consequence of migraine, since migraine is a well-establish disabling condition that may actually limit social and physical capabilities (23). The evidence that emerged from the noticeable number of recent intervention studies seems more solid and reliable. Overall, a general reduction of frequency, severity and duration of migraine attacks has been observed after the exercise intervention periods, and this has also been frequently associated with a considerable improvement of the quality of life of the affected subjects (Table 1). These findings may be of substantial value in a clinical perspective. Severe migraine has a strong and unfavorable impact on the quality of life of the affected patients, since it is a disabling condition which not only impairs social life, but also unfavorably impacts physical functioning (24), often leading to compromised postural control performance (25). This evidence would hence pave the way to hypothesizing the existence of a vicious cycle, in which migraine becomes a major impediment for regular exercise performance and, in turn, a lower 
Table 1 Intervention studies which have explored the putative benefits of physical exercise on migraine

\begin{tabular}{|c|c|c|c|}
\hline Authors & Type of study & Intervention & Outcome \\
\hline Dittrich et al., 2008 (17) & $\begin{array}{l}\text { Randomized, } \\
\text { intervention study }\end{array}$ & $\begin{array}{l}1 \text { hour aerobic exercise program twice } \\
\text { weekly, for } 6 \text { weeks }\end{array}$ & $\begin{array}{l}\text { Reduction of both intense migraine pain and high } \\
\text { frequency of migraine attacks; improvement of } \\
\text { active behavior }\end{array}$ \\
\hline Pairo et al., 2016 (19) & $\begin{array}{l}\text { Randomized, } \\
\text { intervention study }\end{array}$ & $\begin{array}{l}40 \text { min moderate intensity aerobic } \\
\text { exercise } 3 \text { times weekly, for } 1 \text { month }\end{array}$ & $\begin{array}{l}\text { Reduction of frequency, intensity and duration of } \\
\text { migraine attacks }\end{array}$ \\
\hline Varkey et al., 2017 (20) & Intervention study & Double exhaustion cycle ergometer test & $\begin{array}{l}\text { Development of migraine attack in } 57 \% \text { of patients } \\
\text { with a history of exercise-provoked migraine }\end{array}$ \\
\hline Krøll et al., 2018 (22) & $\begin{array}{l}\text { Randomized, } \\
\text { intervention study }\end{array}$ & $\begin{array}{l}45 \text { min of aerobic exercise } 3 \text { times } \\
\text { per week, for } 3 \text { months }\end{array}$ & $\begin{array}{l}\text { Reduction of pain intensity, pain duration and } \\
\text { frequency of migraine attacks; improvement of } \\
\text { both perceived quality of life and physically active } \\
\text { lifestyle }\end{array}$ \\
\hline
\end{tabular}

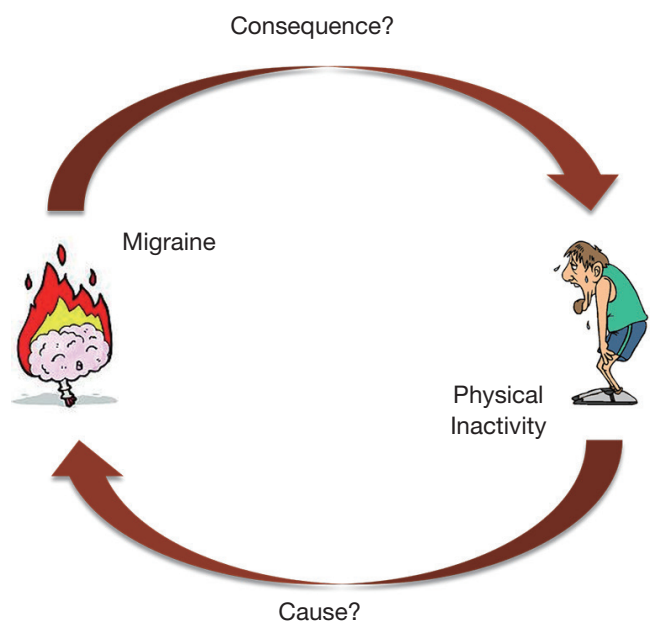

Figure 1 The "vicious cycle" between physical inactivity and migraine.

level of physical exercise would then contribute to worsen severity, frequency and duration of migraine attacks, thus further limiting the willingness of migraineurs to exercise (Figure 1). Therefore, regular engagement in moderateintensity aerobic exercise (i.e., $\geq 40 \mathrm{~min}, 3$ times per week) may be regarded as a reasonable preventive measure in these subjects (Table 2). Many biological effects can be triggered by physical exercise, some of which may have a role in the pathogenesis of migraine. These will probably be mediated by chronic adaptation towards enhanced pain threshold and lower stress as commonly induced by regular physical exercise (26). This recommendation is indirectly supported by the evidence that even physiotherapy interventions (including manual therapy, soft-tissue technique and training) may be effective in reducing frequency and pain intensity of migraine (27). Whilst theoretically straightforward, this advice would not apply to patients with a personal history of exercise-provoked migraine (Table 2). This is due to the tangible risk that physical exercise may trigger or worsen a migraine attack in some patients, a process probably mediated by acute increase of the powerful vasodilation compound nitric oxide (NO) (16), which would then overcome the potential longterm benefits of physical exercise.

\section{Acknowledgements}

None. 
Table 2 Literature-based recommendations on physical activity and migraine

Regular moderate aerobic physical exercise (>40 min, 3 times per week) seems effective to reduce both severity and frequency of migraine attacks

Since exercising may sometimes worsen migraine, be engaged in physical exercise during a migraine attack must be established on an individual basis, according to the personal history of exercise-provoked migraine

High-intensity exercise should be avoided in patients with a history of exercise-provoked migraine

\section{Footnote}

Conflicts of Interest: The authors have no conflicts of interest to declare.

\section{References}

1. Charles A. Migraine. N Engl J Med 2017;377:553-61.

2. Charles A. The pathophysiology of migraine: implications for clinical management. Lancet Neurol 2018;17:174-82.

3. Chu KH, Howell TE, Keijzers G, et al. Acute Headache Presentations to the Emergency Department: A Statewide Cross-sectional Study. Acad Emerg Med 2017;24:53-62.

4. Lindblad M, Hougaard A, Amin FM, et al. Can migraine aura be provoked experimentally? A systematic review of potential methods for the provocation of migraine aura. Cephalalgia 2017;37:74-88.

5. Busch V, Gaul C. Exercise in migraine therapy--is there any evidence for efficacy? A critical review. Headache 2008;48:890-9.

6. Varkey E, Hagen K, Zwart JA, et al. Physical activity and headache: results from the Nord-Trondelag Health Study (HUNT). Cephalalgia 2008;28:1292-7.

7. Milde-Busch A, Blaschek A, Borggrafe I, et al. Associations of diet and lifestyle with headache in high-school students: results from a cross-sectional study. Headache 2010;50:1104-14.

8. Winter AC, Hoffmann W, Meisinger C, et al. Association between lifestyle factors and headache. J Headache Pain 2011;12:147-55.

9. Winsvold BS, Hagen K, Aamodt AH, et al. Headache, migraine and cardiovascular risk factors: the HUNT study. Eur J Neurol 2011;18:504-11.

10. Koppen H, van Veldhoven PL. Migraineurs with exercisetriggered attacks have a distinct migraine. J Headache Pain 2013;14:99.

11. Lebedeva ER, Kobzeva NR, Gilev DV, et al. Factors Associated with Primary Headache According to Diagnosis, Sex, and Social Group. Headache 2016;56:341-56.
12. Krøll LS, Hammarlund CS, Westergaard ML, et al. Level of physical activity, well-being, stress and self-rated health in persons with migraine and co-existing tension-type headache and neck pain. J Headache Pain 2017;18:46.

13. Farris SG, Thomas JG, Abrantes AM, et al. Pain worsening with physical activity during migraine attacks in women with overweight/obesity: A prospective evaluation of frequency, consistency, and correlates. Cephalalgia 2017:333102417747231. [Epub ahead of print]

14. Hagen K, Asberg AN, Stovner L, et al. Lifestyle factors and risk of migraine and tension-type headache. Followup data from the Nord-Trondelag Health Surveys 19951997 and 2006-2008. Cephalalgia 2018:333102418764888. [Epub ahead of print]

15. Luedtke K, Mehnert J, May A. Altered muscle activity during rest and during mental or physical activity is not a trait symptom of migraine - a neck muscle EMG study. J Headache Pain 2018;19:26.

16. Narin SO, Pinar L, Erbas D, et al. The effects of exercise and exercise-related changes in blood nitric oxide level on migraine headache. Clin Rehabil 2003;17:624-30.

17. Dittrich SM, Gunther V, Franz G, et al. Aerobic exercise with relaxation: influence on pain and psychological wellbeing in female migraine patients. Clin J Sport Med 2008;18:363-5.

18. Varkey E, Cider A, Carlsson J, et al. A study to evaluate the feasibility of an aerobic exercise program in patients with migraine. Headache 2009;49:563-70.

19. Pairo Z, Parnow AH, Sari-aslani P, et al. Effect of Moderate Intensity Aerobic Exercise on Migraine Headache Indexes and Quality of Life Improvement in Women with Migraine. Quarterly of Horizon of Medical Sciences 2016;22:291-7.

20. Varkey E, Gruner Svealv B, Edin F, et al. Provocation of Migraine after Maximal Exercise: A Test-Retest Study. Eur Neurol 2017;78:22-7.

21. Hanssen H, Minghetti A, Magon S, et al. Superior Effects of High-Intensity Interval Training vs. Moderate Continuous Training on Arterial Stiffness in Episodic 
Migraine: A Randomized Controlled Trial. Front Physiol 2017;8:1086.

22. Krøll LS, Hammarlund CS, Linde M, et al. The effects of aerobic exercise for persons with migraine and co-existing tension-type headache and neck pain. A randomized, controlled, clinical trial. Cephalalgia 2018:333102417752119. [Epub ahead of print]

23. Mannix S, Skalicky A, Buse DC, et al. Measuring the impact of migraine for evaluating outcomes of preventive treatments for migraine headaches. Health Qual Life Outcomes 2016;14:143.

24. Raggi A, Leonardi M, Bussone G, et al. A 3-month

Cite this article as: Lippi G, Mattiuzzi C, Sanchis-Gomar F. Physical exercise and migraine: for or against? Ann Transl Med 2018;6(10):181. doi: 10.21037/atm.2018.04.15 analysis of disability, quality of life, and disease course in patients with migraine. Headache 2013;53:297-309.

25. Carvalho GF, Bonato P, Florencio LL, et al. Balance Impairments in Different Subgroups of Patients With Migraine. Headache 2017;57:363-74.

26. Koseoglu E, Yetkin MF, Ugur F, et al. The role of exercise in migraine treatment. J Sports Med Phys Fitness 2015;55:1029-36.

27. Luedtke K, Allers A, Schulte LH, et al. Efficacy of interventions used by physiotherapists for patients with headache and migraine-systematic review and metaanalysis. Cephalalgia 2016;36:474-92. 